\title{
ANTIMATROIDS, BETWEENNESS, CONVEXITY
}

\author{
Vašek Chvátal ${ }^{1}$ \\ Canada Research Chair in Combinatorial Optimization, \\ Department of Computer Science and Software Engineering, \\ Concordia University, Montréal, Québec H3G 1M8, Canada
}

\section{Introduction}

Korte and Lovász $[12,13]$ founded the theory of greedoids. These combinatorial structures characterize a class of optimization problems that can be solved by greedy algorithms. In particular, greedoids generalize matroids, introduced earlier by Whitney [16]. Antimatroids, introduced by Dilworth [3] as particular examples of semimodular lattices, make up another class of greedoids.

Antimatroids are related to abstract convexity; let us explain how. Kay and Womble [11] defined a convexity space on a ground set $E$ as a tuple $(E, \mathcal{N})$, where $\mathcal{N}$ is a collection of subsets of $E$ such that $\emptyset \in \mathcal{N}, E \in \mathcal{N}$, and $\mathcal{N}$ is closed under intersections. Members of $\mathcal{N}$ are called convex sets. The convex hull of a subset $X$ of $E$ is defined as the intersection of all convex supersets of $X$ and is denoted by $\tau_{\mathcal{N}}(X)$. Independently of each other, Edelman [6] and Jamison [9] initiated the study of convexity spaces $(E, \mathcal{N})$ with the anti-exchange property

if $X \subseteq E$ and $y, z$ are distinct points outside $\tau_{\mathcal{N}}(X)$,

then at most one of $y \in \tau_{\mathcal{N}}(X \cup\{z\})$ and $z \in \tau_{\mathcal{N}}(X \cup\{y\})$ holds true.

Jointly [7], they proposed to call such convexity spaces convex geometries. An antimatroid is a tuple $(E, \mathcal{F})$ such that $(E,\{E-X: X \in \mathcal{F}\})$ is a convex geometry.

In the present paper, we deal exclusively with finite ground sets. Our starting point are two examples of antimatroids. One of these arises from double shelling of a poset (example 2.4 in Chapter III of the monograph [14]) and the other from simplicial shelling of a triangulated graph (example 2.7 in Chapter III of [14]). Let us describe them in terms of convex geometries.

\footnotetext{
${ }^{1}$ This research was funded by the Canada Research Chairs Program and by the Natural Sciences and Engineering Research Council of Canada.
} 
In the first example, given a partially ordered set $(E, \preceq)$, we let $\mathcal{N}$ consist of all subsets $K$ of $E$ such that

$$
a, c \in K, a \prec b \prec c \Rightarrow b \in K ;
$$

the resulting tuple $(E, \mathcal{N})$ is a convex geometry. In the second example, given an undirected graph with a vertex-set $E$, we let $\mathcal{N}$ consist of all subsets $K$ of $E$ such that

$$
a, c \in K, b \text { is an interior vertex of a chordless path from } a \text { to } c \Rightarrow b \in K \text {. }
$$

The resulting tuple $(E, \mathcal{N})$ is a convexity space, but not necessarily a convex geometry: for instance, take a chordless cycle through four vertices as the graph and consider the convex set $X$ consisting of two adjacent vertices in this graph. Nevertheless, if the graph is triangulated, meaning that it contains no chordless cycle through four or more vertices, then $(E, \mathcal{N})$ is a convex geometry. To elucidate this point, we appeal to a characterization of convex geometries that involves the notion of an extreme point of a convex set $K$, defined as a point $b$ of $K$ such that $K-\{b\}$ is convex.

Nine equivalent characterizations of convex geometries are stated in [9]; equivalence of the following four is proved in [14], Chapter III, Theorem 1.1.

Fact 1. For every convexity space $(E, \mathcal{N})$, the following four propositions are logically equivalent:

(G1) $(E, \mathcal{N})$ has the anti-exchange property.

(G2) If $X \in \mathcal{N}$ and $X \neq E$, then $X \cup\{y\} \in \mathcal{N}$ for some $y$ in $E-X$.

(G3) Every set in $\mathcal{N}$ is the convex hull of its extreme points.

(G4) Every subset $X$ of $E$ contains a unique minimal subset $Y$ such that $\tau_{\mathcal{N}}(Y)=\tau_{\mathcal{N}}(X)$

To see that our second example has property (G3), we invoke a theorem of Dirac [4]. There, a vertex is called simplicial if its neighbours are pairwise adjacent.

Fact 2. For every finite undirected graph $G$, the following three propositions are logically equivalent: 
- $G$ is triangulated.

- Every minimal cutset in $G$ is a clique.

- Every induced subgraph of $G$ either includes two nonadjacent simplicial vertices or is complete.

A corollary of this theorem (stated and proved in [8] as Theorem 3.2) asserts that

every non-simplicial vertex in a triangulated graph

lies on a chordless path between two simplicial vertices;

since the extreme points of $E$ in the second example are precisely the simplicial vertices of $G$, it follows that every point of $E$ lies in the convex hull of at most two extreme points of $E$.

Each of these two examples of convex geometries is constructed through the intermediary of betweenness in the underlying structure. In the first example, we may say that $b$ lies between $a$ and $c$ if, and only if, $a \prec b \prec c$ or $c \prec b \prec a$; in the second example, we may say that $b$ lies between $a$ and $c$ if, and only if, $b$ is an interior vertex of a chordless path from $a$ to $c$; in either case, a subset $K$ of $E$ is convex if and only if

$$
a, c \in K, b \text { lies between } a \text { and } c \Rightarrow b \in K \text {. }
$$

This construction generalizes: as in [1], every ternary relation $\mathcal{B}$ on a finite ground set $E$ defines a convexity space $\left(E, \mathcal{N}_{\mathcal{B}}\right)$ by

$$
\mathcal{N}_{\mathcal{B}}=\{K \subseteq E: a, c \in K,(a, b, c) \in \mathcal{B} \Rightarrow b \in K\}
$$

Our objective is to characterize a nested pair of classes of ternary relations $\mathcal{B}$ on finite ground sets $E$ such that the corresponding classes of convexity spaces $\left(E, \mathcal{N}_{\mathcal{B}}\right)$ consist exclusively of convex geometries, include all convex geometries that arise from double shelling of a poset, and include all convex geometries that arise from simplicial shelling of a triangulated graph. 


\section{Results}

Note that, for every ternary relation $\mathcal{B}$ on a finite ground set, every set $K$ in $\mathcal{N}_{\mathcal{B}}$, and every point $b$ of $K$,

$$
K-\{b\} \in \mathcal{N}_{\mathcal{B}} \Leftrightarrow \text { there are no points } a, c \text { of } K \text { such that }(a, b, c) \in \mathcal{B} \text {. }
$$

This observation allows us to extend the definition of extreme points: given an arbitrary, not necessarily convex, subset $X$ of the ground set and given an arbitrary point $b$ of $X$, we shall say that $b$ is an extreme point of $X$ if, and only if, there are no points $a, c$ of $X$ such that $(a, b, c) \in \mathcal{B}$. The set of all extreme points of $X$ will be denoted by $\operatorname{ex}_{\mathcal{B}}(X)$.

In addition, note that $\mathcal{N}_{\mathcal{B}}$ does not change if $\mathcal{B}$ is made symmetric by including $(c, b, a)$ in $\mathcal{B}$ whenever $(a, b, c)$ is in $\mathcal{B}$; it does not change either if all triples $(b, b, b),(b, b, c),(a, b, b)$ are removed from $\mathcal{B}$. Note also that $\mathcal{N}_{\mathcal{B}}$ includes all singletons $\{a\}$ with $a \in E$ (Kay and Womble [11] designate such convexity spaces $\left.T_{1}\right)$ if and only if $\mathcal{B}$ includes no triple $(a, b, a)$ with $b \neq a$. We will restrict our attention to ternary relations $\mathcal{B}$ such that

$$
(a, b, c) \in \mathcal{B} \Rightarrow(c, b, a) \in \mathcal{B} \text { and } a, b, c \text { are pairwise distinct; }
$$

any such $\mathcal{B}$ will be called a strict betweenness.

Theorem 1. For every strict betweenness $\mathcal{B}$ on a finite ground set $E$, the following two propositions are logically equivalent:

(i) For all subsets $X$ of $E$ and all $x_{1}, x_{2}, x_{3}$ in $X$ such that $\left(x_{1}, x_{2}, x_{3}\right) \in \mathcal{B}$, there are $\bar{x}_{1}, \bar{x}_{3}$ in $\operatorname{ex}_{\mathcal{B}}(X)$ such that $\left(\bar{x}_{1}, x_{2}, \bar{x}_{3}\right) \in \mathcal{B}$.

(ii) $\left(a, b, c_{2}\right),\left(c_{1}, c_{2}, c_{3}\right) \in \mathcal{B} \Rightarrow\left(a, b, c_{1}\right) \in \mathcal{B}$ or $\left(a, b, c_{3}\right) \in \mathcal{B}$ or $\left(c_{1}, b, c_{3}\right) \in \mathcal{B}$.

Following [11], a convexity space is said to have Carathéodory number $d$ if, and only if, $d$ is the smallest positive integer with the following property:

if a point lies in the convex hull of a set $X$,

then it lies in the convex hull of a subset $X^{\prime}$ of $X$ such that $\left|X^{\prime}\right| \leq d$.

Theorem 1 characterizes a class of ternary relations $\mathcal{B}$ on finite ground sets $E$ such that the corresponding class of convexity spaces $\left(E, \mathcal{N}_{\mathcal{B}}\right)$ consists exclusively of convex geometries with Carathéodory number at most 2. However, 
it does not characterize all such relations: for instance, if $E=\left\{a, b, c_{2}, c_{3}\right\}$ and

$$
\mathcal{B}=\left\{\left(a, b, c_{2}\right),\left(c_{2}, b, a\right),\left(a, c_{2}, c_{3}\right),\left(\left(c_{3}, c_{2}, a\right)\right\},\right.
$$

then $\left(E, \mathcal{N}_{\mathcal{B}}\right)$ is a convex geometry with Carathéodory number 2 and yet $\mathcal{B}$ does not satisfy the conditions of Theorem 1 .

Strict order betweenness[15] in a partially ordered set $(E, \preceq)$ is defined by

$$
\mathcal{B}=\left\{(a, b, c) \in E^{3}: a \prec b \prec c \text { or } c \prec b \prec a\right\} ;
$$

monophonic [10], or minimal path [5], betweenness in an undirected graph with a vertex-set $E$ is defined by

$\mathcal{B}=\left\{(a, b, c) \in E^{3}: b\right.$ is an interior vertex of a chordless path from $a$ to $\left.c\right\}$.

Strict order betweenness satisfies condition (ii) of Theorem 1: it is a straightforward exercise to verify that it satisfies the stronger condition

$$
\left(a, b, c_{2}\right),\left(c_{1}, c_{2}, c_{3}\right) \in \mathcal{B} \Rightarrow\left(a, b, c_{1}\right) \in \mathcal{B} \text { or }\left(a, b, c_{3}\right) \in \mathcal{B} .
$$

We shall prove that monophonic betweenness, too, satisfies this stronger condition.

Theorem 2. Let $G$ be a finite triangulated graph and let $a, b, c_{1}, c_{2}, c_{3}$ be vertices of $G$. If

$b$ is an interior vertex of a chordless path between $a$ and $c_{2}$ and $c_{2}$ is an interior vertex of a chordless path between $c_{1}$ and $c_{3}$,

then

$b$ is an interior vertex of a chordless path between a and $c_{1}$, or else $b$ is an interior vertex of a chordless path between a and $c_{3}$.

Theorem 3. For every strict betweenness $\mathcal{B}$ on a finite ground set $E$, the following two propositions are logically equivalent:

(i) For all subsets $X$ of $E$ and all $x_{1}, x_{2}, x_{3}$ in $X$ such that $\left(x_{1}, x_{2}, x_{3}\right) \in \mathcal{B}$, there is an $\bar{x}_{3}$ in $\operatorname{ex}_{\mathcal{B}}(X)$ such that $\left(x_{1}, x_{2}, \bar{x}_{3}\right) \in \mathcal{B}$.

(ii) $\left(a, b, c_{2}\right),\left(c_{1}, c_{2}, c_{3}\right) \in \mathcal{B} \Rightarrow\left(a, b, c_{1}\right) \in \mathcal{B}$ or $\left(a, b, c_{3}\right) \in \mathcal{B}$. 


\section{Proofs}

Our proof of Theorem 1 parallels a proof of the theorem of Dietrich [2] that characterizes antimatroids in terms of circuits (see also Theorem 3.9 in Chapter III of [14]). It begins with a pair of auxiliary results.

Lemma 1. Let $\mathcal{B}$ be a strict betweenness on a finite ground set $E$. If

$$
\left(a, b, c_{2}\right),\left(c_{1}, c_{2}, c_{3}\right) \in \mathcal{B} \Rightarrow\left(a, b, c_{1}\right) \in \mathcal{B} \text { or }\left(a, b, c_{3}\right) \in \mathcal{B} \text { or }\left(c_{1}, b, c_{3}\right) \in \mathcal{B},
$$

then, with $\mathcal{N}=\mathcal{N}_{\mathcal{B}}$,

$$
\tau_{\mathcal{N}}(X)=X \cup\{b \text { : there are } a, c \text { in } X \text { with }(a, b, c) \in \mathcal{B}\}
$$

for all subsets $X$ of $E$.

Proof. Write $X^{\prime}=\{b$ : there are $a, c$ in $X$ with $(a, b, c) \in \mathcal{B}\}$. Since $\tau_{\mathcal{N}}(X)$ is a convex superset of $X$, it is a superset of $X \cup X^{\prime}$; our task reduces to proving that $X \cup X^{\prime}$ is convex. For this purpose, consider an arbitrary $b$ in $E$ such that $(a, b, c) \in \mathcal{B}$ for some $a, c$ in $X \cup X^{\prime}$ : we are going to prove that $b \in X \cup X^{\prime}$.

CASE 1: $a, c \in X$.

In this case, $b \in X^{\prime}$ by definition of $X^{\prime}$.

CASE 2: $a \in X, c \in X^{\prime}$.

By definition of $X^{\prime}$, there are $c_{1}, c_{3}$ in $X$ with $\left(c_{1}, c, c_{3}\right) \in \mathcal{B}$; the hypothesis of the lemma with $c_{2}=c$ guarantees that $\left(c_{1}, b, c_{3}\right) \in \mathcal{B}$ or $\left(a, b, c_{1}\right) \in \mathcal{B}$ or $\left(a, b, c_{3}\right) \in \mathcal{B}$. But then we are back in Case 1 .

CASE 3: $a, c \in X^{\prime}$.

As in Case 2, we find $c_{1}, c_{3}$ in $X$ such that $\left(c_{1}, b, c_{3}\right) \in \mathcal{B}$ or $\left(a, b, c_{1}\right) \in \mathcal{B}$ or $\left(a, b, c_{3}\right) \in \mathcal{B}$. If $\left(c_{1}, b, c_{3}\right) \in \mathcal{B}$, then we are back in Case 1 ; if $\left(a, b, c_{1}\right) \in \mathcal{B}$ or $\left(a, b, c_{3}\right) \in \mathcal{B}$, then we are back in Case 2 .

Lemma 2. Let $\mathcal{B}$ be a strict betweenness on a finite ground set $E$. If

$$
\left(a, b, c_{2}\right),\left(c_{1}, c_{2}, c_{3}\right) \in \mathcal{B} \Rightarrow\left(a, b, c_{1}\right) \in \mathcal{B} \text { or }\left(a, b, c_{3}\right) \in \mathcal{B} \text { or }\left(c_{1}, b, c_{3}\right) \in \mathcal{B},
$$

then $\left(E, \mathcal{N}_{\mathcal{B}}\right)$ is a convex geometry.

Proof. Write $\mathcal{N}=\mathcal{N}_{\mathcal{B}}$. We will show that the convexity space $(E, \mathcal{N})$ has the anti-exchange property. For this purpose, assume the contrary: there are a subset $X$ of $E$ and distinct points $y, z$ outside $\tau_{\mathcal{N}}(X)$ such that $y \in$ 
$\tau_{\mathcal{N}}(X \cup\{z\})$ and $z \in \tau_{\mathcal{N}}(X \cup\{y\})$. Since $y \in \tau_{\mathcal{N}}(X \cup\{z\})-\tau_{\mathcal{N}}(X)$ and $y \neq z$, Lemma 1 guarantees that $\left(x_{y}, y, z\right) \in \mathcal{B}$ for some $x_{y}$ in $X$; similarly, $\left(x_{z}, z, y\right) \in \mathcal{B}$ for some $x_{z}$ in $X$. But then the hypothesis with $a=x_{y}, b=y$, $c_{1}=y, c_{2}=z, c_{3}=x_{z}$ implies that $\left(x_{y}, y, x_{z}\right) \in \mathcal{B}$, and so $y \in \tau_{\mathcal{N}}(X)$, a contradiction.

Proof of Theorem 1. To see that (i) implies (ii), set $X=\left\{a, b, c_{1}, c_{2}, c_{3}\right\}$ and $x_{1}=a, x_{2}=b, x_{3}=c_{2}$ in (i). To show that (ii) implies (i), consider an arbitrary subset $X$ of $E$ and arbitrary $x_{1}, x_{2}, x_{3}$ in $X$ such that $\left(x_{1}, x_{2}, x_{3}\right) \in \mathcal{B}$; let $K$ denote the convex hull of $X$. Since $x_{1}, x_{2}, x_{3} \in K$, we have $x_{2} \notin \operatorname{ex}_{\mathcal{B}}(K)$, but Lemma 2 guarantees that $x_{2}$ belongs to the convex hull of $\operatorname{ex}_{\mathcal{B}}(K)$; now Lemma 1 (with $\operatorname{ex}_{\mathcal{B}}(K)$ in place of $X$ ) provides $\bar{x}_{1}, \bar{x}_{3}$ in $\operatorname{ex}_{\mathcal{B}}(K)$ such that $\left(\bar{x}_{1}, x_{2}, \bar{x}_{3}\right) \in \mathcal{B}$. Finally, Lemma 1 shows that $\operatorname{ex}_{\mathcal{B}}(K) \subseteq X$, and so $\bar{x}_{1}, \bar{x}_{3} \in$ $\operatorname{ex}_{\mathcal{B}}(X)$.

Proof of Theorem 2. Let $P_{1}$ denote the chordless path from $a$ to $c_{2}$ that passes through $b$ and let $P_{2}$ denote the chordless path from $c_{1}$ to $c_{3}$ that passes through $c_{2}$. Proceeding along $P_{1}$ from $a$ to $c_{2}$, we label the vertices consecutively as $v_{1}, v_{2}, \ldots, v_{m}$, so that

$$
a=v_{1}, \quad b=v_{s} \text { for some } s \text { such that } 2 \leq s \leq m-1, \quad c_{2}=v_{m} ;
$$

proceeding along $P_{2}$ from $c_{1}$ to $c_{3}$, we label the vertices consecutively as $w_{1}$, $w_{3}, \ldots, w_{n}$, so that

$$
c_{1}=w_{1}, \quad c_{2}=w_{t} \text { for some } t \text { such that } 2 \leq t \leq n-1, \quad c_{3}=w_{n} .
$$

We claim that

$(\star)$ none of $v_{1}, v_{2}, \ldots, v_{m-2}$ has a neighbour $w_{i}$ with $i<t$ or none of $v_{1}, v_{2}, \ldots, v_{m-2}$ has a neighbour $w_{j}$ with $j>t$.

To justify this claim, assume the contrary: there are edges $v_{k} w_{i}$ and $v_{\ell} w_{j}$ with $k, \ell \leq m-2$ and $i<t, j>t$. Choose them so that $|k-\ell|$ is minimized (we may have $k=\ell$ ) and, subject to this constraint, $i$ is maximized and $j$ is minimized; let $P$ denote the segment of $P_{1}$ that stretches between $v_{k}$ and $v_{\ell}$. Now $v_{k}$ is the only vertex on $P$ that has a neighbour in $\left\{w_{1}, w_{2}, \ldots, w_{t-1}\right\}$ and $v_{\ell}$ is the only vertex on $P$ that has a neighbour in $\left\{w_{t+1}, w_{t+2}, \ldots, w_{n}\right\}$; as $w_{t}=v_{m}$ and $k, \ell \leq m-2$, no vertex on $P$ is adjacent to $w_{t}$ or identical with $w_{t}$. It follows that the paths $P$ and $w_{i} w_{i+1} \ldots w_{j}$ are vertex-disjoint and 
that their union induces a chordless cycle through at least four vertices; this contradiction completes the proof of $(\star)$.

After flipping $P_{2}$ if necessary, $(\star)$ lets us assume that none of $v_{1}, v_{2}, \ldots$, $v_{s-1}$ has a neighbour $w_{i}$ with $i<t$. Since the walk $v_{s} v_{s+1} \ldots v_{m} w_{t-1} \ldots w_{2} w_{1}$ connects $b$ to $c_{1}$, some subset of its vertices induces a chordless path $P$ from $b$ to $c_{1}$; now $b$ is an interior vertex of the chordless path $v_{1} v_{2} \ldots v_{s-1} P$ between $a$ and $c_{1}$.

Proof of Theorem 3. To see that (i) implies (ii), set $X=\left\{a, b, c_{1}, c_{2}, c_{3}\right\}$ and $x_{1}=a, x_{2}=b, x_{3}=c_{2}$ in (i). To show that (ii) implies (i), we shall use induction on $|X|$. If $|X| \leq 2$, then the conclusion is vacuously true. For the induction step, consider arbitrary $x_{1}, x_{2}, x_{3}$ in $X$ such that $\left(x_{1}, x_{2}, x_{3}\right) \in \mathcal{B}$. Setting

$$
Z=\left\{z \in X:\left(x_{1}, x_{2}, z\right) \in \mathcal{B}\right\},
$$

we shall proceed to prove that $Z \cap \operatorname{ex}_{\mathcal{B}}(X) \neq \emptyset$.

First we claim that, with $\mathcal{N}$ a shorthand for $\mathcal{N}_{\mathcal{B}}$ as usual,

- $\tau_{\mathcal{N}}(X-Z) \cap Z=\emptyset$.

To justify this claim, assume the contrary: there is a triple $\left(c_{1}, c_{2}, c_{3}\right)$ in $\mathcal{B}$ such that $c_{1} \in X-Z, c_{2} \in Z, c_{3} \in X-Z$. But then the hypothesis of the theorem is contradicted by $a=x_{1}, b=x_{2}$.

Next, let us write $z^{\prime} \prec z^{\prime \prime}$ if and only if $z^{\prime}, z^{\prime \prime} \in Z$ and there exists a $y$ in $X-Z$ such that $\left(y, z^{\prime}, z^{\prime \prime}\right) \in \mathcal{B}$; note that $z^{\prime} \prec z^{\prime \prime} \Rightarrow z^{\prime} \neq z^{\prime \prime}$. We claim that

- $\prec$ is antisymmetric.

To justify this claim, assume the contrary: there are $z_{1}, z_{2}$ in $Z$ with $z_{1} \prec z_{2}$, $z_{2} \prec z_{1}$. By definition, there are $y_{1}, y_{2}$ in $X-Z$ such that $\left(y_{1}, z_{1}, z_{2}\right) \in \mathcal{B}$ and $\left(y_{2}, z_{2}, z_{1}\right) \in \mathcal{B}$. But then the hypothesis of the theorem is contradicted by $a=y_{1}, b=z_{1}, c_{1}=y_{2}, c_{2}=z_{2}, c_{3}=z_{1}$ : since $\tau_{\mathcal{N}}(X-Z) \cap Z=\emptyset$, we have $\left(y_{1}, z_{1}, y_{2}\right) \notin \mathcal{B}$.

In addition, we claim that

- $\prec$ is transitive.

To justify this claim, consider any $z_{1}, z_{2}, z_{3}$ in $Z$ such that $z_{1} \prec z_{2}$ and $z_{2} \prec z_{3}$. By definition, there are $y_{1}, y_{2}$ in $X-Z$ such that $\left(y_{1}, z_{1}, z_{2}\right),\left(y_{2}, z_{2}, z_{3}\right) \in \mathcal{B}$; as $\tau_{\mathcal{N}}(X-Z) \cap Z=\emptyset$ guarantees that $\left(y_{1}, z_{1}, y_{2}\right) \notin \mathcal{B}$, the hypothesis of the 
theorem with $a=y_{1}, b=z_{1}, c_{1}=y_{2}, c_{2}=z_{2}, c_{3}=z_{3}$ implies $\left(y_{1}, z_{1}, z_{3}\right) \in \mathcal{B}$, and so $z_{1} \prec z_{3}$.

Our set $Z$ is nonempty (it includes $x_{3}$ ) and it is partially ordered by $\prec$. Let $Z_{\max }$ denote the set of its maximal elements. We claim that

- $Z_{\max } \cap \operatorname{ex}_{\mathcal{B}}(Z) \subseteq \operatorname{ex}_{\mathcal{B}}(X)$.

To justify this claim, assume the contrary: there are $a, z_{2}, c$ in $X$ such that $z_{2} \in Z_{\max } \cap \operatorname{ex}_{\mathcal{B}}(Z)$ and $\left(a, z_{2}, c\right) \in \mathcal{B}$. Since $\tau_{\mathcal{N}}(X-Z) \cap Z=\emptyset$, at least one of $a, c$ belongs to $Z$; since $z_{2} \in \operatorname{ex}_{\mathcal{B}}(Z)$, at most one of $a, c$ belongs to $Z$; now symmetry allows us to assume that $a \in X-Z$ and $c \in Z$. But then $z_{2} \prec c$, contradicting the assumption that $z_{2} \in Z_{\max }$.

We shall complete the proof by showing that

- $Z_{\max } \cap \operatorname{ex}_{\mathcal{B}}(Z) \neq \emptyset$.

For this purpose, we rely on the induction hypothesis; note that $|Z|<|X|$ as $Z$ includes neither $x_{1}$ nor $x_{2}$.

CASE 1: $Z_{\max } \neq Z$.

In this case, let $z$ be any maximal element of $Z-Z_{\max }$. Since $z \notin Z_{\max }$, there are a $y$ in $X-Z$ and a $z_{2}$ in $Z_{\max }$ such that $\left(y, z, z_{2}\right) \in \mathcal{B}$. If $z_{2} \in \operatorname{ex}_{\mathcal{B}}(Z)$, then we are done; else there are elements $z_{1}, z_{3}$ of $Z$ such that $\left(z_{1}, z_{2}, z_{3}\right) \in \mathcal{B}$. Now the induction hypothesis applied to $Z$ and $\left(z_{1}, z_{2}, z_{3}\right)$ yields a $\bar{z}_{3}$ in $\operatorname{ex}_{\mathcal{B}}(Z)$ such that $\left(z_{1}, z_{2}, \bar{z}_{3}\right) \in \mathcal{B}$; next, the induction hypothesis applied to $Z$ and $\left(\bar{z}_{3}, z_{2}, z_{1}\right)$ yields a $\bar{z}_{1}$ in $\operatorname{ex}_{\mathcal{B}}(Z)$ such that $\left(\bar{z}_{3}, z_{2}, \bar{z}_{1}\right) \in \mathcal{B}$. The hypothesis of the theorem with $a=y, b=z, c_{1}=\bar{z}_{1}, c_{2}=z_{2}, c_{3}=\bar{z}_{3}$ guarantees that a subscript $i$ in $\{1,3\}$ satisfies $z \prec \bar{z}_{i}$; now maximality of $z$ implies $\bar{z}_{i} \in Z_{\max }$.

CASE 2: $Z_{\max }=Z$.

In this case, our task reduces to proving that $\operatorname{ex}_{\mathcal{B}}(Z) \neq \emptyset$. We may assume that $\operatorname{ex}_{\mathcal{B}}(Z) \neq Z$ (else we are done), and so $\mathcal{B}$ includes a triple $\left(z_{1}, z_{2}, z_{3}\right)$ such that $z_{1}, z_{2}, z_{3}$ are elements of $Z$. But then the induction hypothesis applied to $Z$ and $\left(z_{1}, z_{2}, z_{3}\right)$ yields a $\bar{z}_{3}$ in $\operatorname{ex}_{\mathcal{B}}(Z)$.

\section{References}

[1] J.R. Calder, "Some elementary properties of interval convexities", $J$. London Math. Soc. (2) 3 (1971), 422-428. 
[2] B.L. Dietrich, "A circuit set characterization of antimatroids", J. Combin. Theory Ser. B 43 (1987), 314-321.

[3] R.P. Dilworth, "Lattices with unique irreducible decompositions", Ann. of Math. (2) 41 (1940), 771-777.

[4] G.A. Dirac, "On rigid circuit graphs", Abh. Math. Sem. Univ. Hamburg 25 (1961), 71-76.

[5] P. Duchet, "Convex sets in graphs. II. Minimal path convexity", J. Combin. Theory Ser. B 44 (1988), 307-316.

[6] P.H. Edelman, "Meet-distributive lattices and the anti-exchange closure", Algebra Universalis 10 (1980), 290-299.

[7] P.H. Edelman and R.E. Jamison, "The theory of convex geometries", Geometriae Dedicata 19 (1985), 247-270.

[8] M. Farber and R.E. Jamison, "Convexity in graphs and hypergraphs", SIAM Journal on Algebraic and Discrete Methods 7 (1986), 433-444.

[9] R.E. Jamison-Waldner, "Copoints in antimatroids", Congressus Numerantium 29 (1980), 535-544.

[10] R.E. Jamison-Waldner, "A perspective on abstract convexity: classifying alignments by varieties", in: Convexity and related combinatorial geometry (Norman, Okla., 1980), pp. 113-150, Lecture Notes in Pure and Appl. Math. 76, Dekker, New York, 1982.

[11] D.C. Kay and E.W. Womble, "Axiomatic convexity theory and relationships between the Carathéodory, Helly, and Radon numbers", Pacific Journal of Mathematics 38 (1971), 471-485.

[12] B. Korte and L. Lovász, "Mathematical structures underlying greedy algorithms", in: Fundamentals of computation theory (Szeged, 1981), pp. 205-209, Lecture Notes in Computer Science 117, Springer, BerlinNew York, 1981.

[13] B. Korte and L. Lovász, "Greedoids - a structural framework for the greedy algorithm", in: Progress in combinatorial optimization (Waterloo, Ont., 1982), pp. 221-243, Academic Press, Toronto, ON, 1984. 
[14] B. Korte, L. Lovász, and R. Schrader, Greedoids, Springer-Verlag, Berlin, 1991.

[15] J. Lihová, Strict order-betweennesses, Acta Univ. M. Belii Ser. Math. 8 (2000), 27-33. http://matematika.fpv.umb.sk/papers8.ps

[16] H. Whitney, "On the abstract properties of linear dependence", American Journal of Mathematics 57 (1935), 509-533. 